\title{
MicroRNAs: a great challenge for the diagnosis and therapy of endocrine cancers
}

\author{
Alfredo Fusco
}

Istituto di Endocrinologia ed Oncologia Sperimentale, del CNR c/o Dipartimento di Biologia e Patologia, Cellulare e Molecolare, Facoltá di Medicina e Chirurgia di Napoli, Universitá degli Studi di Napoli 'Federico II' via Pansini 5, 80131 Naples, Italy

(Correspondence should be addressed to A Fusco; Email: afusco@napoli.com, alfusco@ unina.it)

MicroRNAs (miRNAs or miRs) have emerged as an important class of short endogenous RNAs that act as post-transcriptional regulators of gene expression via sequence-specific interactions with the $3^{\prime}$-untranslated regions of cognate mRNA targets. In mammalian cells, miRs affect gene silencing via both mRNA translational inhibition and degradation. One miR is capable of regulating several distinct mRNAs and, altogether, the human miRs identified so far are believed to modulate more than one-third of the mRNA species encoded in the genome (Lewis et al. 2003, John et al. 2004, Kiriakidou et al. 2004). Moreover, each gene may be regulated by more than one miR. Therefore, the potential regulatory circuitry afforded by miRs is enormous.

A central role for miRs in the establishment and progression of human tumors has begun to emerge. More than $50 \%$ of miR-encoding loci reside in chromosomal regions altered during tumorigenesis, and expression profiling reveals characteristic miR signatures for many tumor types that predict disease status and clinical outcome. Moreover, the analysis of several miR targets has evidenced that they are represented by oncogenes or tumor suppressor genes (Calin \& Croce 2006, Volinia et al. 2006).

Because of the great importance of miR deregulation in cancer, Endocrine-Related Cancer is pleased to present a focus issue on the role of miRs in endocrine cancers and in biological mechanisms critical for the acquisition of the malignant phenotype, such as differentiation, apoptosis, and cell invasion. Scientists with great expertise in this field summarize current findings in six reviews.

Santarpia et al. (2010) describe the mechanism by which miRs act during the multiple steps that drive the progressive transformation of normal cells into highly malignant cells. Moreover, they highlight miRs of potential relevance to endocrine tumors and hormonedependent cancers, providing new insights into the development of potential therapies for endocrine tumors based on miR target systems. The review by Coppola et al. (2010) summarizes the current knowledge about $\mathrm{miR}$ expression in prostate cancer, focusing on the $\mathrm{miR}$ targets whose deregulation may have a critical role in prostate carcinogenesis. The possible use of candidate miRs for clinical biochemistry and therapeutics is also taken into consideration. Pallante et al. (2010) summarize the current findings about miR expression in human thyroid carcinomas, focusing on the possible mechanisms by which their deregulation may play a role in carcinogenesis. Moreover, they discuss the possible role of miR profiling as a tool in the diagnosis of thyroid neoplastic diseases, and consider ways in which to exploit information on deregulated miRs to improve thyroid cancer therapeutics. In their review, Boyerinas et al. (2010) summarize the most relevant studies establishing a crucial role for let-7 in normal development and differentiation, and provide an overview of the association between deregulated let-7 expression and tumorigenesis. Boyerinas et al. also review the regulation of let-7 expression, its relevant cancer-involved targets, and the relationship between let-7 and drug sensitivity. Dahiya \& Morin (2010) have summarized the studies that have lead to identification of the miRs deregulated in ovarian cancer: most of them regulate target genes coding for proteins with critical functions in the acquisition of the neoplastic phenotype and may represent targets for cancer therapy. Moreover, they discuss miR expression in ovarian cancer, and their possible role in disease detection, diagnosis, and therapy. In their review, Vecchione \& Croce (2010) focused their attention on the miRs involved in the apoptotic process. The identification and characterization of these miRs may have a great clinical relevance in the near future since most cytotoxic anticancer agents induce apoptosis, and the expression of these miRs may modulate the sensitivity to cytotoxic cancer chemotherapy. Indeed, evasion of apoptosis underlies tumorigenesis and represents a major obstacle to successful therapy. Consequently, miR targeting might 
help to enhance sensitivity to cancer treatment and increase survival.

Our knowledge about miRs in cancer is still incomplete, and there is some inconsistency in the data, which needs to be resolved before diagnostic and/or therapeutic applications can be envisaged. However, the rapid expansion of this field and the extent of the perspectives offered foresee exciting future advances. Moreover, because of the rapid discovery and annotation of new miRs, there is the need to develop new techniques to study their activity. Several bioinformatic tools can be used to predict the binding sites of miRs to their target mRNA. However, only a fraction of miRs are being experimentally confirmed to regulate their predicted mRNA targets. This represents an important methodological challenge in the miR field and, at the moment, represents one of the difficulties in the miR functional annotation.

Therapeutic strategies based on the modulation of miR activity hold great promise due to the ability of these small molecules to potently influence cellular behavior. The first examples of using miR restoration to inhibit carcinogenesis in experimental in vivo models have recently been published. Indeed, $m i R-31$ inhibition using a 'sponge' strategy was recently shown to inhibit breast cancer metastasis in vivo (Valastyan et al. 2009), and restoration of miR-26 expression in hepatocellular carcinoma cells using an adeno-associated virus resulted in the inhibition of cell proliferation, induction of tumor-specific apoptosis, and remarkable protection from disease progression (Kota et al. 2009). Moreover, Elmén et al. (2008) successfully antagonized the hepatocyte expression of $m i R-122$ in nonhuman primates by the simple systemic delivery of locked nucleic acid-modified oligonucleotides (LNA-antimiRs). These findings clearly demonstrate the utility of systemically delivered LNA-antimiRs, supporting their use in the therapy of miR-associated diseases. Therefore, the greatest challenge of future research in this field will be to translate this rapidly expanding knowledge to human cancer therapy.

\section{Declaration of interest}

The author declares that there is no conflict of interest that could be perceived as prejudicing the impartiality of this work.

\section{Funding}

A Fusco receives funding from AIRC, Italian Association for Cancer Research.

\section{References}

Boyerinas B, Park S-M, Hau A, Murmann AE \& Peter ME 2010 The role of let-7 in cell differentiation and cancer. Endocrine-Related Cancer 17 F19-F36.

Calin GA \& Croce CM 2006 MicroRNA signatures in human cancers. Nature Reviews. Cancer 6 857-866.

Coppola V, De Maria R \& Bonci D 2010 MicroRNAs and prostate cancer. Endocrine-Related Cancer 17 F1-F17.

Dahiya V \& Morin PJ 2010 MicroRNAs in ovarian carcinomas. Endocrine-Related Cancer 17 F77-F89.

Elmén J, Lindow M, Schütz S, Lawrence M, Petri A, Obad S, Lindholm M, Hedtjärn M, Hansen HF, Berger U et al. 2008 LNA-mediated microRNA silencing in non-human primates. Nature $\mathbf{4 5 2} 896-899$.

John B, Enright AJ, Aravin A, Tuschl T, Sander C \& Marks DS 2004 Human microRNA targets. PLoS Biology 2 e363.

Kiriakidou M, Nelson PT, Kouranov A, Fitziev P, Bouyioukos C, Mourelatos Z \& Hatzigeorgiou A 2004 A combined computational-experimental approach predicts human microRNA targets. Genes and Development 18 $1165-1178$.

Kota J, Chivukula RR, O’Donnell KA, Wentzel EA, Montgomery CL, Hwang HW, Chang TC, Vivekanandan P, Torbenson M, Clark KR et al. 2009 Therapeutic microRNA delivery suppresses tumorigenesis in a murine liver cancer model. Cell 137 1005-1017.

Lewis BP, Shih IH, Jones-Rhoades MW, Bartel DP \& Burge CB 2003 Prediction of mammalian microRNA targets. Cell 115 787-798.

Pallante P, Visone R, Croce CM \& Fusco A 2010 Deregulation of microRNA expression in follicular cellderived human thyroid carcinomas. Endocrine-Related Cancer 17 F91-F104.

Santarpia L, Nicoloso M \& Calin GA 2010 MicroRNAs: a complex regulatory network drives the acquisition of malignant cell phenotype. Endocrine-Related Cancer 17 F51-F75.

Valastyan S, Reinhardt F, Benaich N, Calogrias D, Szász AM, Wang ZC, Brock JE, Richardson AL \& Weinberg RA 2009 A pleiotropically acting microRNA, miR-31, inhibits breast cancer metastasis. Cell $\mathbf{1 3 7}$ 1032-1046.

Vecchione A \& Croce CM 2010 'Apoptomirs': small molecules have gained the license to kill. EndocrineRelated Cancer 17 F37-F49.

Volinia S, Calin GA, Liu CG, Ambs S, Cimmino A, Petrocca F, Visone R, Iorio M, Roldo C, Ferracin M et al. 2006 A microRNA expression signature of human solid tumors defines cancer gene targets. PNAS $\mathbf{1 0 3}$ 2257-2261. 